\title{
Tight-binding study of stacking fault energies and the Rice criterion of ductility in the fcc metals
}

\author{
Michael J. Mehl and Dimitrios A. Papaconstantopoulos \\ Center for Computational Materials Science, Naval Research Laboratory, Washington, D.C. 20375-5000 \\ Nicholas Kioussis and M. Herbranson \\ Department of Physics and Astronomy, California State University, Northridge California 91330-8268
}

(Received 13 August 1999; revised manuscript received 2 November 1999)

\begin{abstract}
We have used the Naval Research Laboratory (NRL) tight-binding (TB) method to calculate the generalized stacking fault energy and the Rice ductility criterion in the fcc metals $\mathrm{Al}, \mathrm{Cu}, \mathrm{Rh}, \mathrm{Pd}, \mathrm{Ag}, \mathrm{Ir}, \mathrm{Pt}, \mathrm{Au}$, and $\mathrm{Pb}$. The method works well for all classes of metals, i.e., simple metals, noble metals, and transition metals. We compared our results with full potential linear-muffin-tin orbital and embedded atom method (EAM) calculations, as well as experiment, and found good agreement. This is impressive, since the NRL-TB approach only fits to first-principles full-potential linearized augmented plane-wave equations of state and band structures for cubic systems. Comparable accuracy with EAM potentials can be achieved only by fitting to the stacking fault energy.
\end{abstract}

\section{INTRODUCTION}

The relationship between electronic structure and mechanical properties of materials has received considerable attention, with emphasis on the development of atomistic models of fracture and deformation. ${ }^{1}$ The interfacial energetics under different modes of displacement, e.g., shear and cleavage, are often key factors in determining the mechanical response of a material to applied stress. The stability of stacking faults on the slip planes of a crystal is intimately connected to the mobility of dislocations on these planes. On the other hand, the ease for cleavage fracture is related to the ideal cleavage stress and the ideal cleavage energy (which is equal to the total surface energy $\gamma_{s}$ of the two cleaved surface planes). Using a Peierls type of analysis, Rice and coworkers $^{2-4}$ developed a simple criterion for determining the intrinsic ductile versus brittle behavior of materials. Rice proposed that a simple rule to measure the brittle versus ductile behavior of materials is the ratio of two planar fault energies, $\gamma_{s} / \gamma_{u s}$, which determines the competition between dislocation emission from a crack tip and crack cleavage. Dislocation nucleation is characterized by the unstable stacking fault energy $\gamma_{u s}$, which corresponds to the lowest energy barrier encountered in sliding one half of a crystal relative to another along a slip plane.

In close-packed structures, both stable and unstable stacking faults are produced by the relative translation of two parts of a crystal through a fault vector $\vec{f}$, which is a rational fraction of a lattice vector. The fault is introduced by cutting a perfect crystal block along the fault plane and shifting the upper part with respect to the lower part by the vector $\vec{f}$. The energy surface obtained as a function of the fault vector $\vec{f}$ is called the generalized stacking fault (GSF) energy surface. ${ }^{5}$ Stable intrinsic stacking faults are local energy minima on the GSF surface. For the (111) slip plane in fcc systems, the stable stacking fault configuration corresponds to a slip of $a / \sqrt{6}$ in the $\langle 121\rangle$ direction resulting in the stacking $A B C \mid B C A B C ; \gamma_{u s}$ is the lowest energy barrier that needs to be crossed for the slip from the ideal configuration to the intrinsic stacking fault in the $\langle 121\rangle$ direction. Experimentally observed edge dislocations in fcc metals split and form an intrinsic stacking fault ribbon bounded by the two Shockley partials. The intrinsic stacking fault energy, $\gamma_{i s}$, determines the equilibrium separation between the two partials. ${ }^{6}$ In contrast to the rest of the GSF surface, the energies of the stable stacking fault configurations can be experimentally measured.

The GSF surface plays an important role in proposed models for the brittle-ductile transition ${ }^{7,8}$ and they can also be used for calibration of model potentials for large-scale simulations and as input to quasi-continuum models. ${ }^{9}$ At present, the GSF energies can be calculated using empirical potentials, such as the embedded-atom method (EAM) or electronic structure methods. ${ }^{10,11}$ The empirical potentials are expedient but they are constructed by fitting physical properties obtained from experimental measurements or ab initio calculations. Moreover, these potentials may provide a good description of the energetics of a system when the density distribution of atoms in the system is close to equilibrium. When atoms move far away from equilibrium (such as in the case of stress) or when defects are present, these potentials meet their limitations. For example, the current formulation of the EAM method underestimates $\gamma_{i s}$ in $\mathrm{Al}$ compared to the corresponding $a b$ initio value, thus yielding a dissociation for the edge dislocation in disagreement both with experiment and the $a b$ initio calculations. ${ }^{10}$ An improved EAM value for $\gamma_{i s}$ can be obtained by including this energy in the EAM database. ${ }^{10,11}$ The more accurate first-principles electronic structure calculations, on the other hand, are computationally expensive. We would therefore like to find a method that is close in accuracy to first-principles methods, but computationally more efficient.

The NRL tight-binding method ${ }^{12-16}$ meets this criterion for a large number of systems. The purpose of this work is to employ the tight-binding (TB) total-energy method to calculate the GSF energies for fcc metals and to compare them both with results of $a b$ initio calculations based on the fullpotential linear-muffin-tin-orbital (FLMTO) method ${ }^{17,18}$ and experiment, when available. The fcc metals studied have a 
wide range of unstable and stable stacking fault energy values. For example, $\mathrm{Al}$ and $\mathrm{Cu}$ have similar $\gamma_{u s}$ but quite different $\gamma_{i s}$, while Ir (a brittle system) has very large $\gamma_{u s}$ and $\gamma_{i s}$. We have also calculated the ratio $\gamma_{s} / \gamma_{u s}$ across the series. Overall, the good agreement found between the TB results and those of the $a b$ initio calculations indicates that the TB method can be used for the study of fault energies accurately and expediently, and puts the TB method on firmer basis for future investigations of defects and fracture.

\section{STACKING FAULT CALCULATIONS}

We model the $\langle 121\rangle$ slip on a (111) slip plane of an fcc solid by constructing a supercell which consists of nine close-packed (111) planes of atoms. One atom in each plane is part of the basis of the supercell. The primitive vectors of the supercell take the form

$$
\begin{aligned}
& \mathbf{a}_{1}=\frac{1}{2} a \hat{y}+\frac{1}{2} a \hat{z} \\
& \mathbf{a}_{2}=\frac{1}{2} a \hat{x}+\frac{1}{2} a \hat{z} \\
& \mathbf{a}_{3}=\left(3+\frac{q}{6}\right) a \hat{x}+\left(3+\frac{q}{6}\right) a \hat{y}-\left(3-\frac{q}{3}\right) a \hat{z},
\end{aligned}
$$

where $q$ represents the stacking fault variable, and represents a displacement of the atoms in the boundary layer along the fault vector $\vec{f}$ in the $\langle 11 \overline{2}\rangle$ direction. When $q=0$ the periodic crystal is a perfect fcc system. When $q=1$, the atoms at the interface are in an hcp ordering, that is, the stacking at the interface is $A B C B C A$ rather than $A B C A B C$.

Total energies were calculated using the NRL tightbinding (TB) total energy method. ${ }^{12-16,19}$ In this method we simultaneously fit the energy bands and the total energy from first-principles full potential linearized augmented planewave (LAPW) calculations ${ }^{20,21}$ to a nonorthogonal spd TB Hamiltonian containing density-dependent on-site terms and distance-dependent hopping and overlap parameters. The parameters for the transition and noble metals are determined by requiring that the TB method reproduce the firstprinciples total energies and electronic structures of fcc, and $\mathrm{bcc}$ as a function of volume. For $\mathrm{Pb}$ (Ref. 16) we add the simple cubic structure to the database, and for $\mathrm{Al}$ (Ref. 14) we included data from the simple cubic, diamond, and hcp structures. This method has been shown to give reliable structural behavior, elastic constants, phonon frequencies, ${ }^{12}$ vacancy formation energies, and surface energies for the fcc metals, even for structures not included in the original database. We use the previously published parameters ${ }^{13,16}$ for most elements. We have developed new parameters for $\mathrm{Cu}$ and $\mathrm{Au}$ by including the simple cubic structure in the fit along with the fcc and bcc structures, and by fitting to a wider range of lattice constants. ${ }^{22}$ The parameters for $\mathrm{Al}$ differ from the published parameters ${ }^{14}$ in that we have refit the parameters so that the $d$ state $t_{2 g}$ and $e_{g}$ on-site terms are identical, eliminating orientation effects seen in the older parameters.

As in all band structure total energy methods, the calculated total energy is determined by summing the eigenvalues

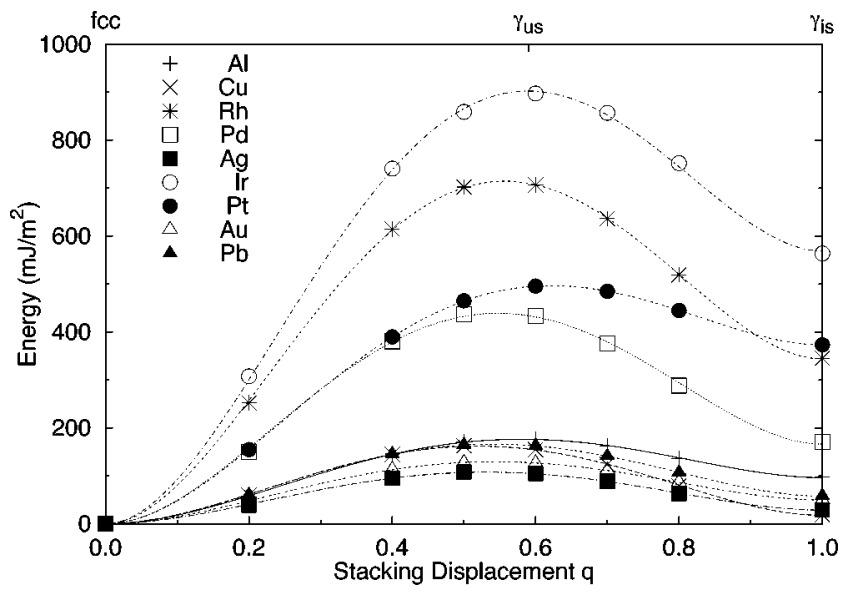

FIG. 1. Stacking fault energy as a function of the parameter $q$ in Eq. (1) for the elemental $f c c$ metals, determined by the NRL tightbinding method (Refs. 12-16 and 19). The labels $\gamma_{u s}$ and $\gamma_{i s}$ indicate positions of the unstable and intrinsic stacking faults, respectively, while $f c c$ indicates the position of the ground state structure.

over the first Brillouin zone of the lattice. We perform this calculation using a regular, uniformly spaced, and symmetrized $k$-point mesh, including the origin. The tight-binding method is computationally very efficient, so to insure convergence we have used a large number of $k$ points, 4730 in the irreducible part of the Brillouin zone of Eq. (1). This is equivalent to using a mesh of $1469 k$ points in the irreducible Brillouin zone of the fcc lattice. The total energy was calculated by weighting the eigenvalues with a Fermi distribution at a temperature of $5 \mathrm{mRy}$ and then extrapolating to zero temperature. ${ }^{23,24}$

In Fig. 1 we show the stacking fault energies for all of our calculations as a function of the stacking fault variable $q$ in Eq. (1). We note that the behavior of the stacking fault energies is quite reasonable. That is, the stacking fault energies for Ir are much larger than the corresponding energies for $\mathrm{Au}$. We did not allow for relaxation in these systems, except for the case of the unstable stacking fault energy of Au and Ir. In this case, the relaxation reduced the unstable stacking fault energy by about $30 \%$ and $40 \%$, respectively.

We then compared the results of the tight-binding parametrization to first-principles FLMTO calculations for Al, Ag, and Ir, using the same stacking fault geometry. In the FLMTO method no shape approximation is made to the potential and the charge density. ${ }^{17,18}$ The basis set, charge density, and potential are expanded in cubic harmonics inside non-overlapping muffin-tin spheres and in Fourier series in the interstitial region. Spherical harmonic expansions were carried through $l_{\max }=8$ for the bases. Spin-orbit coupling was included self consistently. The volume in the muffin-tin spheres radius was kept constant for all deformations to make the basis set as consistent as possible in calculating energies for different deformations. Exchange and correlation were treated in the local-density approximation using the Ceperly and Alder ${ }^{25}$ exchange-correlation functional as parametrized by Perdew and Zunger. ${ }^{26}$ The calculations are performed at the theoretically determined in-plane lattice constant to eliminate the externally imposed stress associated with a nonequilibrium lattice constant. Multiple sets of energy parameters and tail parameters were used. The lower 


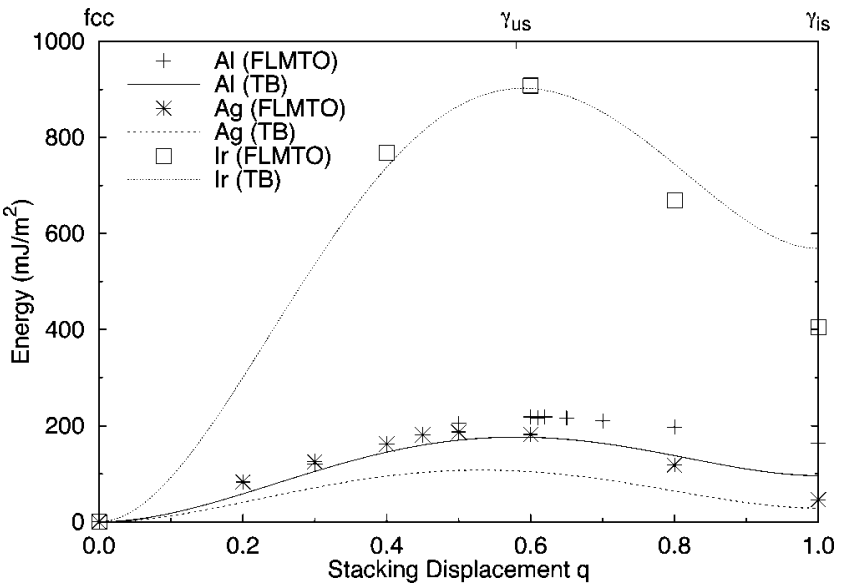

FIG. 2. Stacking fault energy as a function of the parameter $q$ of Eq. (1), as determined by the NRL tight-binding method (Refs. 12-16 and 19) and from first-principles FLMTO calculations (Refs. 17 and 18). Tight-binding results are represented by the fitted curves from Fig. 1, and the first-principles results are marked by points.

energy is appropriate for semicore states and the higher for valence states. For the reciprocal space integration we used a uniform $k$-point grid of $(15,15,3)$ divisions along the reciprocal lattice directions according to the special point method. ${ }^{27}$

Figure 2 compares the first-principles and tight-binding calculations. We see that the tight-binding results are qualitatively correct, that is, the stacking fault energies of the elements are ordered correctly. The results for Ir are particularly accurate, especially near the unstable stacking fault position. In addition, because of the reduced basis-set size and the elimination of the self-consistency cycle, the tightbinding method is approximately 1,000 times faster than FLMTO for comparable systems. Thus the tight-binding method can handle much larger systems than a firstprinciples calculation, with nearly the same accuracy.

The intrinsic ( $q=1$ in equation 1$)$ stacking fault energies $\gamma_{i s}$ are given in Table I, where they are compared to values derived from experimental observations of dislocation structure and isotropic elasticity theory. ${ }^{28,29}$ Again, the results are qualitatively correct.

TABLE I. Intrinsic stacking fault energies $\gamma_{i s}$ (in $\mathrm{mJ} / \mathrm{m}^{2}$ ) of the nonmagnetic fcc transition metals, calculated from our tight-binding parameters, first-principles FLMTO (Refs. 17 and 18), and values derived from experiment. Unless otherwise noted, the experimental values are from Murr (Ref. 28).

\begin{tabular}{cccc}
\hline \hline Element & Tight-Binding & FLMTO & Experiment \\
\hline $\mathrm{Al}$ & 96 & 164 & 166 \\
$\mathrm{Cu}$ & 18 & & 45 (Ref. 28), 78 (Ref. 29) \\
$\mathrm{Rh}$ & 344 & & \\
$\mathrm{Pd}$ & 166 & & 16 \\
$\mathrm{Ag}$ & 29 & 46 & 300 \\
$\mathrm{Ir}$ & 569 & 405 & \\
$\mathrm{Pt}$ & 373 & & \\
$\mathrm{Au}$ & 50 & & \\
$\mathrm{~Pb}$ & 58 & & \\
\hline \hline
\end{tabular}

TABLE II. The unrelaxed (111) surface energy $\gamma_{s}$, unstable stacking fault energy $\gamma_{u s}$, and the ductility parameter $D$ from Eq. (2). The values of $\gamma_{s}$ were obtained from previous calculations using our tight-binding parameters (Refs. 13 and 14). The $\gamma_{u s}$ were taken from the maximum of a polynomial fit to the points in Fig. 1. $\gamma_{s}$ and $\gamma_{u s}$ are in units of $\mathrm{mJ} / \mathrm{m}^{2}$.

\begin{tabular}{cccc}
\hline \hline Element & $\gamma_{s}$ (Refs. 13 and 14) & $\gamma_{u s}$ & $D$ \\
\hline $\mathrm{Al}$ & 870 & 176 & 1.50 \\
$\mathrm{Cu}$ & 1730 & 162 & 3.24 \\
$\mathrm{Rh}$ & 2460 & 714 & 1.04 \\
$\mathrm{Pd}$ & 1570 & 439 & 1.08 \\
$\mathrm{Ag}$ & 1140 & 108 & 3.20 \\
$\mathrm{Ir}$ & 2590 & 902 & 0.87 \\
$\mathrm{Pt}$ & 2510 & 497 & 1.53 \\
$\mathrm{Au}$ & 1480 & 129 & 3.48 \\
$\mathrm{~Pb}$ & 888 & 166 & 1.59 \\
\hline \hline
\end{tabular}

\section{INTRINSIC DUCTILITY}

Rice $^{3}$ has shown that under "Mode I" loading a metal is intrinsically ductile if the parameter

$$
D=0.3 \frac{\gamma_{s}}{\gamma_{u s}}>1,
$$

where $\gamma_{s}$ is the surface energy of a freshly cleaved surface, and $\gamma_{u s}$ is the unstable stacking fault energy. This is a reasonable criterion. If the surface energy is small compared to the unstable stacking fault energy then under applied stress the metal will tend to form surfaces, i.e., crack, rather than shear so as to nucleate dislocations.

For our purposes we can take the unstable stacking fault energy $\gamma_{u s}$ to be the peak of the curve in Fig. 1. For surface energies, we take the unrelaxed (111) surface energies previously computed from our tight-binding parameters. ${ }^{13,14}$ The results are shown in Table II. Since we have neglected relaxation of both the surface and the stacking fault the results can be only qualitatively correct, but the results are consistent with our expectations. The three noble metals, $\mathrm{Cu}$, $\mathrm{Ag}$, and $\mathrm{Au}$, all have $D>3$. Of these, Au, the most ductile metal, has the largest ductility parameter. These are followed by $\mathrm{Pt}, \mathrm{Pb}$, and $\mathrm{Al}$, all with $D \approx 1.5$. These metals are considerably less ductile than the noble metals, but substantially more ductile than the remaining metals, $\mathrm{Rh}, \mathrm{Pd}$, and Ir, which all have $D \approx 1$. These results indicate that the tightbinding Hamiltonian can be used to quickly estimate the ductility parameter of a metal.

\section{CONCLUSION}

We have shown that the NRL tight-binding method is very successful in predicting both intrinsic and unstable stacking fault energies of the fcc metals on the right-hand side of the periodic table. This occurs without the introduction of any stacking fault configurations into the tightbinding database. Indeed, excepting Aluminum, the tightbinding parameters are determined by fitting to a firstprinciples database containing only electronic structure and total energy information for cubic systems. In contrast, the successful EAM schemes ${ }^{10,11}$ must include $\gamma_{i s}$ in the database in order to correctly predict the stacking fault energies. 


\section{ACKNOWLEDGMENTS}

M.J.M. and D.A.P. are supported by the U.S. Office of Naval Research. The development of the static code was supported in part by the U.S. Department of Defense Common HPC Software Support Initiative (CHSSI). The research at California State University Northridge was supported by the U.S. Army Grants Nos. DAAH0495 and DAAG5597. This work was supported in part by a grant of HPC time from the DoD HPC Center, for computations on the IBM SP2 and SGI Origin at the Aeronautical Systems Center, Wright-Patterson Air Force Base, Dayton, $\mathrm{OH}$.
${ }^{1}$ J. R. Rice, G. E. Beltz, and Y. Sun, in Topics in Fracture and Fatigue, edited by A. S. Argon (Springer Verlag, Berlin, 1992).

${ }^{2}$ J. R. Rice and R. M. Thomson, Philos. Mag. 29, 73 (1974).

${ }^{3}$ J. R. Rice, J. Mech. Phys. Solids 40, 239 (1992).

${ }^{4}$ J. R. Rice and G. E. Beltz, J. Mech. Phys. Solids 42, 333 (1994).

${ }^{5}$ V. Vitek, Philos. Mag. 18, 773 (1968); Cryst. Lattice Defects 5, 1 (1974).

${ }^{6}$ J. P. Hirth and J. Lothe, Theory of Dislocations, 2nd ed. (Wiley, New York, 1982).

${ }^{7}$ E. Kaxiras and M. S. Duesbery, Phys. Rev. Lett. 70, 3752 (1993).

${ }^{8}$ S. J. Zhou, A. E. Carlsson, and R. Thomson, Phys. Rev. Lett. 72, 852 (1994)

${ }^{9}$ B. E. Tadmor, M. Ortiz, and R. Phillips, Philos. Mag. A 73, 1529 (1996).

${ }^{10}$ G. Lu, N. Kioussis, V. Bulatov, and E. Kaxiras, Philos. Mag. (to be published)

${ }^{11}$ Y. Mishin, D. Farkas, M. J. Mehl, and D. A. Papaconstantopoulos, Phys. Rev. B 59, 3393 (1999).

${ }^{12}$ R. E. Cohen, M. J. Mehl, and D. A. Papaconstantopoulos, Phys. Rev. B 50, R14 694 (1994).

${ }^{13}$ M. J. Mehl and D. A. Papaconstantopoulos, Phys. Rev. B 54, 4519 (1996) (transition metal parameters).

${ }^{14}$ S. H. Yang, M. J. Mehl, and D. A. Papaconstantopoulos, Phys. Rev. B 57, R2013 (1998). (Al parameters).

${ }^{15}$ M. J. Mehl and D. A. Papaconstantopoulos, in Topics in Compu- tational Materials Science, edited by C. Y. Fong (World Scientific, Singapore, 1998), Chap. V.

${ }^{16}$ D. A. Papaconstantopoulos, M. J. Mehl, and B. Akdim (unpublished).

${ }^{17}$ David L. Price, John M. Wills, and Bernard R. Cooper, Phys. Rev. B 48, 15301 (1993).

${ }^{18}$ S. Sun, N. Kioussis, and M. Ciftan, Phys. Rev. B 54, 3074 (1996).

${ }^{19}$ Tight-binding parameters and codes can be found at http://cstwww.nrl.navy.mil/bind/dodtb/

${ }^{20}$ O. K. Andersen, Phys. Rev. B 12, 3060 (1975).

${ }^{21}$ S. H. Wei and H. Krakauer, Phys. Rev. Lett. 55, 1200 (1985).

${ }^{22}$ The new parameters for $\mathrm{Cu}$ and $\mathrm{Au}$ are available at http://cst-www.nrl.navy.mil/bind/cu_par_99 and http://cstwww.nrl.navy.mil/bind/au_par_99, respectively. A paper discussing the behavior of these parameters under a variety of circumstances will be published in the future.

${ }^{23}$ M. J. Gillan, J. Phys.: Condens. Matter 1, 689 (1989).

${ }^{24}$ O. Grotheer and M. Fähnle, Phys. Rev. B 58, 13459 (1998).

${ }^{25}$ D. M. Ceperly and B. J. Alder, Phys. Rev. Lett. 45, 566 (1980).

${ }^{26}$ J. P. Perdew and A. Zunger, Phys. Rev. B 23, 5048 (1981).

${ }^{27}$ D. J. Chadi and M. L. Cohen, Phys. Rev. B 8, 5747 (1973).

${ }^{28}$ L. E. Murr, Interfacial Phenomena in Metals and Alloys (Addison Wesley, Reading, MA, 1975).

${ }^{29}$ C. Carter and I. Ray, Philos. Mag. 35, 1161 (1977). 